\title{
Suita conjecture and the Ohsawa-Takegoshi extension theorem
}

\section{Zbigniew Błocki}

Received: 3 April 2012 / Accepted: 26 August 2012 / Published online: 8 September 2012 (C) The Author(s) 2012. This article is published with open access at Springerlink.com

Abstract We prove a conjecture of N. Suita which says that for any bounded domain $D$ in $\mathbb{C}$ one has $c_{D}^{2} \leq \pi K_{D}$, where $c_{D}(z)$ is the logarithmic capacity of $\mathbb{C} \backslash D$ with respect to $z \in D$ and $K_{D}$ the Bergman kernel on the diagonal. We also obtain optimal constant in the Ohsawa-Takegoshi extension theorem.

\section{Introduction}

Suita [16] (see also [15, p. 179]) conjectured that for a bounded domain $D$ in $\mathbb{C}$ one has

$$
c_{D}^{2} \leq \pi K_{D}
$$

Here

$$
c_{D}(z)=\exp \left(\lim _{\zeta \rightarrow z}\left(G_{D}(\zeta, z)-\log |\zeta-z|\right)\right)
$$

is the logarithmic capacity of the complement of $D$ with respect to $z \in D$, where $G_{D}(\cdot, z)$ the (negative) Green function for $D$ with pole at $z$, and

$$
K_{D}(z)=\sup \left\{|f(z)|^{2}: f \text { holomorphic in } D, \int_{D}|f|^{2} d \lambda \leq 1\right\}
$$

\footnotetext{
Z. Błocki (凶)

Instytut Matematyki, Uniwersytet Jagielloński, Łojasiewicza 6, 30-348 Kraków, Poland e-mail: Zbigniew.Blocki@im.uj.edu.pl

Z. Błocki

e-mail: umblocki@cyf-kr.edu.pl
} 
denotes the Bergman kernel on the diagonal. One can easily show that we have equality in (1) if $D$ is simply connected. Suita in [16], using the theory of elliptic functions, proved strict inequality in (1) when $D$ is an annulus, and hence also any regular doubly connected domain.

This conjecture has geometric interpretation and consequences. Suita also observed that

$$
K_{D}=\frac{1}{\pi} \frac{\partial^{2}}{\partial z \partial \bar{z}}\left(\log c_{D}\right)
$$

and thus that (1) is equivalent to the fact that the curvature of the invariant metric $c_{D}|d z|$ is bounded above by -4 . Therefore (1) means precisely that maximal curvature is attained on the boundary (if $D$ is smooth then one can show that this curvature is equal to -4 on $\partial D$, or equivalently that the ratio $c_{D}^{2} / \pi K_{D}$ is equal to 1 there). This is not the case for other invariant metrics related to the Bergman kernel, e.g. $K_{D}|d z|^{2}$ and the Bergman metric $\left(\log K_{D}\right)_{z \bar{z}}|d z|^{2}$ (see [9] and [19]). On the other hand, this property is satisfied for the Carathéodory metric (see [17] and [18]) but this is much simpler to prove.

The relation of the Suita conjecture to the extension theorem was first observed and explored by Ohsawa [12]: to prove (1) for a given $z \in D$ we have to find a holomorphic $f$ in $D$ with $f(z)=1$ and

$$
\int_{D}|f|^{2} d \lambda \leq \frac{\pi}{\left(c_{D}(z)\right)^{2}}
$$

Using methods developed in [14] he proved the estimate

$$
c_{D}^{2} \leq C \pi K_{D}
$$

with $C=750$. This was later improved to $C=2$ in [5], where the main tool was an estimate from [1]. A slightly better constant $C=1.954$ was recently obtained in [10], see also [6] for a much simpler proof.

Our goal is to show the following version of the Ohsawa-Takegoshi theorem with optimal constant which in particular implies the Suita conjecture:

Theorem 1 Assume that $\Omega \subset \mathbb{C}^{n-1} \times D$ is pseudoconvex, where $D$ is a bounded domain in $\mathbb{C}$ containing the origin. Then for any holomorphic $f$ in $\Omega^{\prime}:=\Omega \cap\left\{z_{n}=0\right\}$ and $\varphi$ plurisubharmonic in $\Omega$ one can find a holomorphic extension $F$ of $f$ to $\Omega$ with

$$
\int_{\Omega}|F|^{2} e^{-\varphi} d \lambda \leq \frac{\pi}{\left(c_{D}(0)\right)^{2}} \int_{\Omega^{\prime}}|f|^{2} e^{-\varphi} d \lambda^{\prime} .
$$


This kind of result, with a worse constant though, was first formulated in [8] (it used the proof of the Ohsawa-Takegoshi theorem from [2]), see also [13].

Our proof is in big part motivated by the recent work of Chen [7] who showed that the Ohsawa-Takegoshi theorem can deduced directly from the classical Hörmander estimate for the $\bar{\partial}$-equation [11]. We use Chen's idea in the proof of Theorem 2 below. It improves a related $\bar{\partial}$-estimate from [6] which gave Theorem 1 but only with the same constant as in [10]. This $\bar{\partial}$-estimate was one of the crucial improvements compared to [6]. Another one was the solution of the following ODE problem: find $g \in C^{0,1}\left(\mathbb{R}_{+}\right), h \in C^{1,1}\left(\mathbb{R}_{+}\right)$ such that $h$ is convex and decreasing, $g+\log t, h+\log t$ vanish at $\infty$, and

$$
\left(1-\frac{\left(g^{\prime}\right)^{2}}{h^{\prime \prime}}\right) e^{2 g-h+t} \geq 1
$$

The author would like to express his gratitude to Takeo Ohsawa for his constant interest and encouragement throughout the years and to Peter Pflug for essentially introducing him to the theory of Bergman kernel back in 1998.

\section{The proof}

Similarly as in [7] our main tool will be the Hörmander estimate: if $\Omega$ is pseudoconvex in $\mathbb{C}^{n}$ and

$$
\alpha=\sum_{j} \alpha_{j} d \bar{z}_{j} \in L_{l o c,(0,1)}^{2}(\Omega)
$$

is such that $\bar{\partial} \alpha=0$ then for any smooth, strongly plurisubharmonic $\varphi$ in $\Omega$ one can find $u \in L_{l o c}^{2}(\Omega)$ such that

$$
\bar{\partial} u=\alpha
$$

and

$$
\int_{\Omega}|u|^{2} e^{-\varphi} d \lambda \leq \int_{\Omega}|\alpha|_{i \partial \bar{\partial} \varphi}^{2} e^{-\varphi} d \lambda
$$

Here

$$
|\alpha|_{i \partial \bar{\partial} \varphi}^{2}=\sum_{j, k} \varphi^{j \bar{k}} \bar{\alpha}_{j} \alpha_{k}
$$

(where $\left(\varphi^{j \bar{k}}\right)$ is the inverse transposed of the complex Hessian $\left(\partial^{2} \varphi / \partial z_{j} \partial \bar{z}_{k}\right)$ ) denotes the length of $\alpha$ with respect to the Kähler metric $i \partial \bar{\partial} \varphi$. 
It was observed in [4] that (2) makes also sense (and indeed remains true) for arbitrary plurisubharmonic $\varphi$ : instead of $|\alpha|_{i \partial \bar{\partial} \varphi}^{2}$ one should take any $H \in L_{l o c}^{\infty}(\Omega)$ with

$$
i \bar{\alpha} \wedge \alpha \leq H i \partial \bar{\partial} \varphi .
$$

In this convention we formulate our new estimate for the $\bar{\partial}$-equation:

Theorem 2 Let $\alpha \in L_{\text {loc, }(0,1)}^{2}(\Omega)$ be a $\bar{\partial}$-closed form in a pseudoconvex domain $\Omega$ in $\mathbb{C}^{n}$. Assume that $\varphi$ is plurisubharmonic in $\Omega, \psi \in W_{\text {loc }}^{1,2}(\Omega)$ is locally bounded from above and they satisfy $|\bar{\partial} \psi|_{i \partial \bar{\partial} \varphi}^{2} \leq H<1$ in $\Omega$ for some $H \in L_{l o c}^{\infty}(\Omega)$. Then there exists $u \in L_{\text {loc }}^{2}(\Omega)$ solving $\bar{\partial} u=\alpha$ and such that for every $b>0$

$$
\int_{\Omega}|u|^{2}(1-H) e^{2 \psi-\varphi} d \lambda \leq\left(1+\frac{1}{b}\right) \int_{\Omega}|\alpha|_{i \partial \bar{\partial} \varphi}^{2} \frac{1+b H}{1-H} e^{2 \psi-\varphi} d \lambda .
$$

If in addition $H \leq \delta<1$ on $\operatorname{supp} \alpha$ then

$$
\int_{\Omega}|u|^{2}(1-H) e^{2 \psi-\varphi} d \lambda \leq \frac{1+\sqrt{\delta}}{1-\sqrt{\delta}} \int_{\Omega}|\alpha|_{i \partial \bar{\partial} \varphi}^{2} e^{2 \psi-\varphi} d \lambda
$$

Proof By standard approximation we may assume that $\varphi$ is smooth up to the boundary, strongly plurisubharmonic, and that $\psi$ is bounded in $\Omega$. Note that then $L^{2}\left(\Omega, e^{\psi-\varphi}\right), L^{2}\left(\Omega, e^{-\varphi}\right)$ and $L^{2}(\Omega)$ are the same sets. We use a trick from [3]: define $u$ to be the minimal solution to $\bar{\partial} u=\alpha$ in $L^{2}\left(\Omega, e^{\psi-\varphi}\right)$ which means that $u$ is perpendicular to ker $\bar{\partial}$ there. Then $v:=u e^{\psi}$ is perpendicular to $\operatorname{ker} \bar{\partial}$ in $L^{2}\left(\Omega, e^{-\varphi}\right)$ and thus it is the minimal solution to $\bar{\partial} v=\beta$, where

$$
\beta:=e^{\psi}(\alpha+u \bar{\partial} \psi) \in L_{l o c,(0,1)}^{2}(\Omega) .
$$

By Hörmander's estimate (2)

$$
\int_{\Omega}|v|^{2} e^{-\varphi} d \lambda \leq \int_{\Omega}|\beta|_{i \partial \bar{\partial} \varphi}^{2} e^{-\varphi} d \lambda
$$

which means that

$$
\begin{aligned}
\int_{\Omega}|u|^{2} e^{2 \psi-\varphi} d \lambda & \leq \int_{\Omega}|\alpha+u \bar{\partial} \psi|_{i \partial \bar{\partial} \varphi}^{2} e^{2 \psi-\varphi} d \lambda \\
& \leq \int_{\Omega}\left(|\alpha|_{i \partial \bar{\partial} \varphi}^{2}+2|u| \sqrt{H}|\alpha|_{i \partial \bar{\partial} \varphi}+|u|^{2} H\right) e^{2 \psi-\varphi} d \lambda .
\end{aligned}
$$


For $t>0$ we will get

$$
\begin{aligned}
& \int_{\Omega}|u|^{2}(1-H) e^{2 \psi-\varphi} d \lambda \\
& \quad \leq \int_{\Omega}\left[|\alpha|_{i \partial \bar{\partial} \varphi}^{2}\left(1+t^{-1} \frac{H}{1-H}\right)+t|u|^{2}(1-H)\right] e^{2 \psi-\varphi} d \lambda .
\end{aligned}
$$

We obtain the first estimate if we take $t:=1 /(b+1)$, whereas the second one follows if we let $b:=\delta^{-1 / 2}$.

Proof of Theorem 1 Using appropriate approximation we may assume that $\Omega$ is bounded, smooth and strongly pseudoconvex, $\varphi$ is smooth up to the boundary and that $f$ is holomorphic in a neighborhood of $\overline{\Omega^{\prime}}$. Fix $\varepsilon>0$ and define

$$
\alpha:=\bar{\partial}\left(f\left(z^{\prime}\right) \chi\left(-2 \log \left|z_{n}\right|\right)\right)=-\frac{f\left(z^{\prime}\right) \chi^{\prime}\left(-2 \log \left|z_{n}\right|\right)}{\bar{z}_{n}} d \bar{z}_{n},
$$

where we write $z=\left(z^{\prime}, z_{n}\right)$ and $\chi \in C^{0,1}(\mathbb{R})$ is non-decreasing and such that $\chi=0$ on $\{t \leq-2 \log \varepsilon\}, \chi(\infty)=1$ (it will be precisely determined later). Note that $\alpha$ is defined in $\Omega$ for sufficiently small $\varepsilon$, supp $\alpha \subset\left\{\left|z_{n}\right| \leq \varepsilon\right\}$, and for any solution of $\bar{\partial} u=\alpha$ the function

$$
F:=f\left(z^{\prime}\right) \chi\left(-2 \log \left|z_{n}\right|\right)-u
$$

is a holomorphic extension of $f$ provided that $u=0$ on $\Omega^{\prime}$.

In order to use Theorem 2 and define appropriate weights $\tilde{\varphi}, \psi$ the choice of the following two functions on $\mathbb{R}_{+}$is absolutely crucial:

$$
\begin{aligned}
& h(t):=-\log \left(t+e^{-t}-1\right), \\
& g(t):=-\log \left(t+e^{-t}-1\right)+\log \left(1-e^{-t}\right) .
\end{aligned}
$$

They solve the ODE problem formulated in the introduction: one can check that they are decreasing, convex,

$$
\left(1-\frac{\left(g^{\prime}\right)^{2}}{h^{\prime \prime}}\right) e^{2 g-h+t}=1
$$

and

$$
\lim _{t \rightarrow \infty}\left(h(t)-2 g(t)+\log \left(-h^{\prime}(t)\right)\right)=0 .
$$

(In fact, as noticed by the referee, we have $g=\log \left(-h^{\prime}\right)$. This substitution reduces (5) to

$$
\frac{d^{2}}{d t^{2}}\left(e^{-h}\right)=e^{-t}
$$


and enables to solve the desired ODE problem immediately.)

For $G:=G_{D}(\cdot, 0)$ we can write $G=\log |\zeta|+v$, where $v$ is a bounded harmonic function in $D$. There are positive constants $C_{0}, C_{1}$ such that

$$
|2 G-2 \log | \zeta|| \leq C_{0} \quad \text { in } D
$$

and

$$
\left|2 G_{\zeta}-\frac{1}{\zeta}\right| \leq C_{1} \text { near the origin. }
$$

For $M:=-2 \log \varepsilon-C_{0}$ we define

$$
\eta(t):= \begin{cases}h(t), & t<M, \\ -\delta \log (t-M+a)+b, & t \geq M\end{cases}
$$

and

$$
\gamma(t):= \begin{cases}g(t), & t<M, \\ -\delta \log (t-M+a)+\widetilde{b}, & t \geq M,\end{cases}
$$

where $\delta=M^{-1 / 2}$ and $a, b, \widetilde{b}$ are chosen in such a way that $\eta \in C^{1,1}\left(\mathbb{R}_{+}\right)$and $\gamma \in C^{0,1}\left(\mathbb{R}_{+}\right)$. Then

$$
\begin{aligned}
& a=a(\varepsilon)=-\frac{\delta}{h^{\prime}(M)}, \\
& b=b(\varepsilon)=h(M)+\delta \log a, \\
& \widetilde{b}=\widetilde{b}(\varepsilon)=g(M)+\delta \log a .
\end{aligned}
$$

Note that $\delta$ is selected so that

$$
\lim _{\varepsilon \rightarrow 0} \delta(\varepsilon)=0, \quad \lim _{\varepsilon \rightarrow 0} a(\varepsilon)=\infty .
$$

Now we are ready to define

$$
\tilde{\varphi}:=\varphi+2 G+\eta(-2 G), \quad \psi:=\gamma(-2 G) .
$$

Then $\tilde{\varphi}$ is plurisubharmonic in $\Omega$ and $\psi \in W_{l o c}^{1,2}(D)$ is locally bounded from above. We have

$$
|\bar{\partial} \psi|_{i \partial \bar{\partial} \widetilde{\varphi}}^{2} \leq|\bar{\partial} \psi|_{i \partial \bar{\partial}(\eta(-2 G))}^{2}=\frac{\left(\gamma^{\prime}(-2 G)\right)^{2}}{\eta^{\prime \prime}(-2 G)} \begin{cases}<1 & \text { in } \Omega, \\ =\delta & \text { on } \operatorname{supp} \alpha\end{cases}
$$


(note that $\operatorname{supp} \alpha \subset\{-2 G \geq M\}$ ) and

$$
|\alpha|_{i \partial \bar{\partial} \tilde{\varphi}}^{2} \leq|\alpha|_{i \partial \bar{\partial}(\eta(-2 G))}^{2}=\frac{\left|f\left(z^{\prime}\right)\right|^{2}\left(\chi^{\prime}\left(-2 \log \left|z_{n}\right|\right)\right)^{2}}{4\left|G_{z_{n}}\right|^{2}\left|z_{n}\right|^{2} \eta^{\prime \prime}(-2 G)},
$$

where we follow the convention discussed before Theorem 2. Since the function $-\delta \log (t-M+a)+t$ is increasing in $t$ by (5) we have

$$
\left(1-\frac{\left(\gamma^{\prime}\right)^{2}}{\eta^{\prime \prime}}\right) e^{2 \gamma-\eta+t} \begin{cases}=1 & \text { on }\{t<M\}, \\ \geq(1-\delta) e^{2 g(M)-h(M)+M} & \text { on }\{t \geq M\}\end{cases}
$$

and thus for sufficiently small $\varepsilon$

$$
\left(1-\frac{\left(\gamma^{\prime}\right)^{2}}{\eta^{\prime \prime}}\right) e^{2 \gamma-\eta+t} \geq 1
$$

on $\mathbb{R}_{+}$. Theorem 2 now gives a solution $u=u_{\varepsilon}$ of $\bar{\partial} u=\alpha$ such that

$$
\int_{\Omega}|u|^{2} e^{-\varphi} d \lambda \leq \int_{\Omega}|u|^{2}\left(1-|\bar{\partial} \psi|_{i \partial \bar{\partial} \widetilde{\varphi}}^{2}\right) e^{2 \psi-\widetilde{\varphi}} d \lambda \leq \frac{1+\sqrt{\delta}}{1-\sqrt{\delta}} \mathcal{A}(\varepsilon),
$$

where

$$
\mathcal{A}(\varepsilon):=\int_{\Omega} \frac{\left|f\left(z^{\prime}\right)\right|^{2}\left(\chi^{\prime}\left(-2 \log \left|z_{n}\right|\right)\right)^{2}}{4\left|G_{z_{n}}\right|^{2}\left|z_{n}\right|^{2} \eta^{\prime \prime}(-2 G)} e^{2 \psi-\widetilde{\varphi}} d \lambda
$$

We have

$$
\varlimsup_{\varepsilon \rightarrow 0} \mathcal{A}(\varepsilon) \leq \varlimsup_{\varepsilon \rightarrow 0} \mathcal{B}(\varepsilon) \int_{\Omega^{\prime}}|f|^{2} e^{-\varphi} d \lambda^{\prime}
$$

where

$$
\mathcal{B}(\varepsilon):=\int_{\{|\zeta| \leq \varepsilon\}} \frac{\left(\chi^{\prime}(-2 \log |\zeta|)\right)^{2}}{4\left|G_{\zeta}\right|^{2}|\zeta|^{2} \eta^{\prime \prime}(-2 G)} e^{2 \psi-\eta(-2 G)-2 G} d \lambda(\zeta) .
$$

We now want to replace $G$ by $\log |\zeta|$ in the above integral. Note that

$$
\lim _{\varepsilon \rightarrow 0} \sup _{\{|\zeta| \leq \varepsilon\}}|\zeta|^{2} e^{-2 G}=\frac{1}{\left(c_{D}(0)\right)^{2}}
$$

and near the origin

$$
4\left|G_{\zeta}\right|^{2}|\zeta|^{2} \geq\left(1-C_{1}|\zeta|\right)^{2}
$$

Moreover on $\{|\zeta| \leq \varepsilon\}$ we have

$$
2 \psi-\eta(-2 G) \leq 2 \gamma\left(-2 \log |\zeta|-C_{0}\right)-\eta\left(-2 \log |\zeta|-C_{0}\right)
$$




$$
\leq 2 \gamma(-2 \log |\zeta|)-\eta(-2 \log |\zeta|)+\delta \log \left(1+\frac{C_{0}}{a}\right)
$$

and

$$
\eta^{\prime \prime}(-2 G) \geq \eta^{\prime \prime}\left(-2 \log |\zeta|+C_{0}\right) \geq\left(\frac{a+C_{0}}{a+2 C_{0}}\right)^{2} \eta^{\prime \prime}(-2 \log |\zeta|) .
$$

Hence, from (7) it follows that

$$
\varlimsup_{\varepsilon \rightarrow 0} \mathcal{B}(\varepsilon) \leq \frac{1}{\left(c_{D}(0)\right)^{2}} \varlimsup_{\varepsilon \rightarrow 0} \widetilde{\mathcal{B}}(\varepsilon)
$$

where

$$
\begin{aligned}
\widetilde{\mathcal{B}}(\varepsilon) & =\int_{\{|\zeta| \leq \varepsilon\}} \frac{\left(\chi^{\prime}(-2 \log |\zeta|)\right)^{2}}{|\zeta|^{2} \eta^{\prime \prime}(-2 \log |\zeta|)} e^{2 \gamma(-2 \log |\zeta|)-\eta(-2 \log |\zeta|)} d \lambda(\zeta) \\
& =\pi \int_{M+C_{0}}^{\infty} \frac{\left(\chi^{\prime}\right)^{2} e^{2 \gamma-\eta}}{\eta^{\prime \prime}} d t .
\end{aligned}
$$

By the Schwarz inequality the optimal choice of increasing $\chi$ with

$$
\chi(\infty)=\int_{M+C_{0}}^{\infty} \chi^{\prime} d t=1
$$

is

$$
\chi(t):= \begin{cases}0, & t<M+C_{0}, \\ \frac{1}{c} \int_{M+C_{0}}^{t} w d s, & t \geq M+C_{0},\end{cases}
$$

where $w=\eta^{\prime \prime} e^{\eta-2 \gamma}$ and $c=\int_{M+C_{0}}^{\infty} w d t$. Then

$$
\widetilde{\mathcal{B}}(\varepsilon)=\frac{\pi}{\int_{M+C_{0}}^{\infty} \eta^{\prime \prime} e^{\eta-2 \gamma} d t}
$$

and

$$
\begin{aligned}
\int_{M+C_{0}}^{\infty} \eta^{\prime \prime} e^{\eta-2 \gamma} d t & =\delta e^{b-2 \widetilde{b}} \int_{C_{0}}^{\infty}(t+a)^{\delta-2} d t \\
& =\frac{1}{1-\delta}\left(1+\frac{C_{0}}{a}\right)^{\delta-1} e^{h(M)-2 g(M)+\log \left(-h^{\prime}(M)\right)}
\end{aligned}
$$

By (6) and (7)

$$
\lim _{\varepsilon \rightarrow 0} \widetilde{\mathcal{B}}(\varepsilon)=\pi
$$


and we have thus obtained that

$$
\varlimsup_{\varepsilon \rightarrow 0} \int_{\Omega}\left|u_{\varepsilon}\right|^{2} e^{-\varphi} d \lambda \leq \frac{\pi}{\left(c_{D}(0)\right)^{2}} \int_{\Omega^{\prime}}|f|^{2} e^{-\varphi} d \lambda^{\prime} .
$$

Finally, we note that if $0<\widetilde{\varepsilon} \leq \varepsilon$ then

$$
\begin{aligned}
& \int_{\{|\zeta| \leq \widetilde{\varepsilon}\}}\left(1-\frac{\left(\gamma^{\prime}(-2 G)\right)^{2}}{\eta^{\prime \prime}(-2 G)}\right) e^{2 \psi-\eta(-2 G)-2 G} d \lambda(\zeta) \\
& \geq \frac{1}{C(\varepsilon)} \int_{\{|\zeta| \leq \widetilde{\varepsilon}\}} \frac{e^{2 \gamma(-2 \log |\zeta|)-\eta(-2 \log |\zeta|)}}{|\zeta|^{2}} d \lambda(\zeta) \\
& \quad=\frac{\pi e^{2 \widetilde{b}-b}}{C(\varepsilon)} \int_{-2 \log \widetilde{\varepsilon}}^{\infty}(t-M+a)^{-\delta} d t \\
& =\infty
\end{aligned}
$$

and (8) implies that $u=0$ almost everywhere on $\Omega^{\prime}$. The weak limit of $F=F_{\varepsilon}$ given by (4) is the required extension.

Open Access This article is distributed under the terms of the Creative Commons Attribution License which permits any use, distribution, and reproduction in any medium, provided the original author(s) and the source are credited.

\section{References}

1. Berndtsson, B.: Weighted estimates for $\bar{\partial}$ in domains in $\mathbb{C}$. Duke Math. J. 66, 239-255 (1992)

2. Berndtsson, B.: The extension theorem of Ohsawa-Takegoshi and the theorem of Donnelly-Fefferman. Ann. Inst. Fourier 46, 1083-1094 (1996)

3. Berndtsson, B.: Weighted estimates for the $\bar{\partial}$-equation. In: Complex Analysis and Geometry, Columbus, OH, 1999. Ohio State Univ. Math. Res. Inst. Publ., vol. 9, pp. 43-57. de Gruyter, Berlin (2001)

4. Błocki, Z.: The Bergman metric and the pluricomplex Green function. Trans. Am. Math. Soc. 357, 2613-2625 (2005)

5. Błocki, Z.: Some estimates for the Bergman kernel and metric in terms of logarithmic capacity. Nagoya Math. J. 185, 143-150 (2007)

6. Błocki, Z.: On the Ohsawa-Takegoshi extension theorem. http://gamma.im.uj.edu.pl/ blocki (2012)

7. Chen, B.-Y.: A simple proof of the Ohsawa-Takegoshi extension theorem. arXiv:1105. 2430v1

8. Dinew, Ż.: The Ohsawa-Takegoshi extension theorem on some unbounded sets. Nagoya Math. J. 188, 19-30 (2007)

9. Dinew, Ż.: An example for the holomorphic sectional curvature of the Bergman metric. Ann. Pol. Math. 98, 147-167 (2010)

10. Guan, Q., Zhou, X., Zhu, L.: On the Ohsawa-Takegoshi $L^{2}$ extension theorem and the Bochner-Kodaira identity with non-smooth twist factor. J. Math. Pures Appl. 97, 579-601 (2012) 
11. Hörmander, L.: $L^{2}$ estimates and existence theorems for the $\bar{\partial}$ operator. Acta Math. 113, 89-152 (1965)

12. Ohsawa, T.: Addendum to "On the Bergman kernel of hyperconvex domains". Nagoya Math. J. 137, 145-148 (1995)

13. Ohsawa, T.: On the extension of $L^{2}$ holomorphic functions $\mathrm{V}$-effects of generalization. Nagoya Math. J. 161, 1-21 (2001)

14. Ohsawa, T., Takegoshi, K.: On the extension of $L^{2}$ holomorphic functions. Math. Z. 195, 197-204 (1987)

15. Sario, L., Oikawa, K.: Capacity Functions. Grundlehren Math. Wiss., vol. 149. Springer, Berlin (1969)

16. Suita, N.: Capacities and kernels on Riemann surfaces. Arch. Ration. Mech. Anal. 46, 212-217 (1972)

17. Suita, N.: On a metric induced by analytic capacity. Kodai Math. Semin. Rep. 25, 215-218 (1973)

18. Wong, B.: On the holomorphic curvature of some intrinsic metrics. Proc. Am. Math. Soc. 65, 57-61 (1977)

19. Zwonek, W.: Asymptotic behavior of the sectional curvature of the Bergman metric for annuli. Ann. Pol. Math. 98, 291-299 (2010) 